Bull. Austral. Math. Soc.

53A55, 53С40, 14 н 99

Vol. 44 (1991) [483-499]

\title{
HOLOMORPHIC CURVES IN THE ORTHOGONAL TWISTOR SPACE
}

\section{ANDY Talmadge and KICHOON Yang}

A complete description of holomorphic curves in the Hermitian symmetric space $S O(6) / U(3)$ is given in terms of orthogonal differential invariants.

\section{INTRODUCTION}

The Hermitian symmetric space $S O(2 n) / U(n)$ is naturally identified with the twistor space $\mathcal{T}\left(\mathbb{R}^{2 n}\right)$, the space of orthogonal complex structures on $\mathbb{R}^{2 n}$. Thus a curve in $S O(2 n) / U(n)$ can be thought of as a 1-parameter family of complex structures on $\mathbb{R}^{2 n}$, and the study of holomorphic curves in $S O(2 n) / U(n)$ pertains to the deformation problem of complex structures.

The case of $S O(6) / U(3)$ is particularly appealing as this space is symmetric space isomorphic to $\mathbb{C} P^{3}$, albeit via a complicated isomorphism. (The space $S O(4) / U(2)$ is symmetric space isomorphic to $\mathbb{C} P^{1}$, and the geometry of curves in $S O(4) / U(2)$ is trivial.) The study of holomorphic curves in $S O(6) / U(3)$ yields a new perspective on the study of holomorphic curves, and more generally minimal surfaces, in $\mathrm{CP}^{3}$ (see [2]).

In the present paper we give a complete description of holomorphic curves in $S O(6) / U(3)$ in terms of orthogonal differential invariants. Given a Riemann surface $M$ we derive a system of partial differential equations with 3 unknown functions, $\left(\tau_{i}\right)$, on $M$. These partial differential equations (Section $4(\mathrm{I} 1-3)$ ) are the integrability conditions in the following sense: given a solution $\left(\tau_{i}\right)$ one can manufacture a holomorphic curve by integrating a Frobenius system. To put it another way, a solution to the integrability conditions determines a holomorphic curve constructively up to integration involving ordinary differential equations only. Moreover, every holomorphic curve in $S O(6) / U(3)$ arises in this manner.

\section{ThE SPACE OF ORTHOGONAL COMPLEX STRUCTURES ON $\mathbb{R}^{2 n}$}

Let $\mathcal{T}\left(\mathbb{R}^{2 n}\right)$ denote the space of orientation preserving orthogonal complex structures on $\mathbb{R}^{2 n}$. More precisely,

$$
\mathcal{T}\left(\mathbb{R}^{2 n}\right)=\left\{J \in \operatorname{Aut}^{+}\left(\mathbb{R}^{2 n}\right): J^{2}=-i d,\langle J v, J w\rangle=\langle v, w\rangle, v, w \in \mathbb{R}^{2 n}\right\},
$$

Received 13 December 1990

This research was supported in part by an ASU faculty research grant.

Copyright Clearance Centre, Inc. Serial-fee code: 0004-9729/91 \$A2.00+0.00. 
where Aut ${ }^{+}\left(\mathbb{R}^{2 n}\right)$ denotes the set of orientation preserving automorphisms of $\mathbb{R}^{2 n}$. The space $\mathcal{T}\left(\mathbb{R}^{2 n}\right)$ can be identified with the Hermitian symmetric space $S O(2 n) / U(n)$ as we shall see below.

Let $i: U(n) \rightarrow S O(2 n)$ be the Lie group monomorphism induced by the identification

$$
\mathbb{R}^{2 n}=\mathbb{C}^{n},\left(x^{\alpha}\right) \leftrightarrow\left(x^{1}+i x^{2}, \cdots, x^{2 n-1}+i x^{2 n}\right) .
$$

More explicitly, if one writes

$$
\begin{gathered}
Z=X+i Y \in U(n), X, Y \text { real matrices, } \\
i(Z)=S\left[\begin{array}{rr}
X & -Y \\
Y & X
\end{array}{ }^{t} S\right.
\end{gathered}
$$

then

where ${ }^{t} S=\left(\varepsilon_{1}, \varepsilon_{n+1}, \varepsilon_{2}, \varepsilon_{n+2}, \cdots, \varepsilon_{n}, \varepsilon_{2 n}\right)$ and $\varepsilon_{i}$ denotes the column vector with 1 at the $i$ th entry and zeros elsewhere.

We put $H=i(U(n)) \subset S O(2 n)$; we shall occasionally confuse $H$ with $U(n)$. Let $A=\left(A_{\alpha}\right)=\left(A_{1}, \ldots, A_{2 n}\right) \in S O(2 n)$, and consider the assignment

$$
A \mapsto J_{A} \in \mathcal{T}\left(\mathbb{R}^{2 n}\right)
$$

given by the prescription

$$
J_{A}\left(A_{2 i-1}\right)=A_{2 i}, J_{A}\left(A_{2 i}\right)=-A_{2 i-1}, 1 \leqslant i \leqslant n,
$$

that is, the matrix of $J_{A}$ with respect to the basis $\left(A_{\alpha}\right)$ is

$$
j_{n}=\left[\begin{array}{rrrrrrr}
0 & -1 & & & & \\
1 & 0 & & & & \\
& & \cdot & & & & \\
& & & & & \\
& & & & 0 & -1 \\
& & & & 1 & 0
\end{array}\right] .
$$

This assignment induces a bijection

$$
S O(2 n) / H \rightarrow T\left(\mathbb{R}^{2 n}\right)
$$

as it is easily seen that $J_{A}=J_{B}$ if and only if the cosets $A H$ and $B H$ coincide.

In what follows we recall the root space decomposition of the Lie algebra $o(2 n)$. First define $\varepsilon_{\beta}^{\alpha}$ to be the $2 n \times 2 n$ with 1 at the $(\alpha, \beta)$-entry and zeros elsewhere. We 
then define the following matrices in $o(2 n)(o(2 n)$ is thought of as the set of all $2 n \times 2 n$ real skew-symmetric matrices):

$$
\begin{aligned}
F_{i} & =\varepsilon_{2 i-1}^{2 i}-\varepsilon_{2 i}^{2 i-1}, \\
E_{i j} & =\varepsilon_{2 j-1}^{2 i-1}+\varepsilon_{2 j}^{2 i}-\varepsilon_{2 i-1}^{2 j-1}-\varepsilon_{2 i}^{2 j}, \\
F_{i j} & =\varepsilon_{2 j-1}^{2 i}+\varepsilon_{2 i-1}^{2 j}-\varepsilon_{2 i}^{2 j-1}-\varepsilon_{2 j}^{2 i-1}, \\
E_{i j}^{\prime} & =\varepsilon_{2 j-1}^{2 i-1}+\varepsilon_{2 i}^{2 j}-\varepsilon_{2 i-1}^{2 j-1}-\varepsilon_{2 j}^{2 i}, \\
F_{i j}^{\prime} & =\varepsilon_{2 j}^{2 i-1}+\varepsilon_{2 j-1}^{2 i}-\varepsilon_{2 i-1}^{2 j}-\varepsilon_{2 i}^{2 j-1},
\end{aligned}
$$

where $1 \leqslant i<j \leqslant n$. Now put

$$
V_{i}=\mathbb{R}-\operatorname{span}\left\{F_{i}\right\}, \quad V_{i j}=\mathbb{R}-\operatorname{span}\left\{E_{i j}, F_{i j}\right\}, \quad V_{i j}^{\prime}=\mathbb{R}-\operatorname{span}\left\{E_{i j}^{\prime}, F_{i j}^{\prime}\right\}
$$

The root spaces of $\sigma(2 n)$ relative to the standard maximal torus

$$
T=S O(2)^{n} \subset S O(2 n)
$$

$\left(S O(2)^{n}\right.$ is diagonally included in $\left.S O(2 n)\right)$ are precisely

$$
\oplus \sum V_{i}=t, V_{i j}, V_{i j}^{\prime}
$$

where $t$ is the Lie algebra of $T$, the trivial root space.

The Lie algebra of $H \subset S O(2 n)$ is given by

so that

$$
\mathfrak{h}=\left\{X \in \mathfrak{o}(2 n):{ }^{t} X j_{n}+j_{n} X=0\right\}
$$

$$
h=t \oplus \sum V_{i j} \text {. }
$$

Consequently

$$
m=\oplus \sum V_{i j}^{\prime}
$$

is the orthogonal complement to $h$ relative to the Killing form. Via $\pi_{* e}$, where $\pi$ is the projection $S O(2 n) \rightarrow S O(2 n) / H, m$ gets identified with the tangent space at the identity coset of $S O(2 n) / H$ :

$$
\mathrm{m}=T_{0}(S O(2 n) / H), \quad 0=H
$$

Let $\Omega=\left(\Omega_{\beta}^{\alpha}\right)$ denote the o(2n)-valued Maurer-Cartan form of $S O(2 n)$. We then have the decomposition

$$
\Omega=\Omega_{\mathfrak{h}}+\Omega_{\mathrm{m}}, \quad \Omega_{\mathrm{m}}=\sum \Omega_{V_{i j}^{\prime}}
$$


where

$$
\Omega_{V_{i j}^{\prime}}=\frac{1}{2}\left[\left(\Omega_{2 j-1}^{2 i-1}-\Omega_{2 j}^{2 i}\right) \otimes E_{i j}^{\prime}+\left(\Omega_{2 j-1}^{2 i}+\Omega_{2 j}^{2 i-1}\right) \otimes F_{i j}^{\prime}\right] \text { (no sum). }
$$

We also put

$$
\Theta^{\prime i j}=\frac{1}{2}\left[\left(\Omega_{2 j-1}^{2 i-1}-\Omega_{2 j}^{2 i}\right)+\sqrt{-1}\left(\Omega_{2 j-1}^{2 i}+\Omega_{2 j}^{2 i-1}\right)\right], \quad 1 \leqslant i<j \leqslant n .
$$

Recall that the space $S O(2 n) / U(n)$ has, up to conjugacy, exactly one integrable almost complex structure; this complex structure is characterised by letting the pullbacks of $\left(\Theta^{\prime} i j\right)$ (by a local section of $S O(2 n) \rightarrow S O(2 n) / H$ ) span the space of type $(1,0)$ forms on $S O(2 n) / H$.

Any invariant metric on $S O(2 n) / H$ is given by the pullback of the symmetric product

$$
c \cdot \sum \Theta^{\prime i j} \cdot \bar{\Theta}^{\prime i j}, c>0 .
$$

We shall use the metric coming from $c=1$.

\section{THE LINEAR ISOTROPY REPRESENTATION}

In this section we explicitly compute the linear isotropy representation

$$
\rho: U(n) \rightarrow G L(\mathrm{~m})=G L\left(T_{0}(S O(2 n) / H)\right) .
$$

For $Z \in U(n)$ one has the inner automorphism

$$
\operatorname{Inn}_{z}: S O(2 n) \rightarrow S O(2 n), g \mapsto z y z^{-1},
$$

where $z=i(Z) \in H$. The map $\operatorname{Inn}_{z}$ fixes the origin $0=H \in S O(2 n) / H$ and we obtain the automorphism

$$
\operatorname{Inn}_{z * 0}: m \rightarrow m, m=T_{0}(S O(2 n) / H) .
$$

The assignment

$$
\left.Z \in U(n) \mapsto \operatorname{Ad}(Z)\right|_{m}=\operatorname{Inn}_{z * 0} \in G L(m)
$$

is nothing but the adjoint representation of $U(n)$ restricted to the invariant subspace $\mathrm{m} \subset \mathrm{o}(2 n)(\operatorname{Ad}(H) \mathrm{m} \subset \mathrm{m})$. Then $\rho=\operatorname{Ad} \mid \mathrm{m}$.

In what follows we take $n=3$. Recall that a basis of $m$ is given by

$$
E_{12}^{\prime}, E_{13}^{\prime}, E_{23}^{\prime}, F_{12}^{\prime}, F_{13}^{\prime}, F_{23}^{\prime} \in \mathrm{o}(6) \text {. }
$$


$\operatorname{Ad}_{Z}\left(E_{i j}^{\prime}\right)$ is computed from the matrix multiplication

$$
i(Z) \cdot E_{i j}^{\prime} \cdot i(Z)^{-1}
$$

Written out more fully,

$$
\operatorname{Ad}_{Z}\left(E_{i j}^{\prime}\right)=\left(S \cdot\left[\begin{array}{rr}
X & -Y \\
Y & X
\end{array}\right] \cdot{ }^{t} S\right) E_{i j}^{\prime}\left(S \cdot\left[\begin{array}{cc}
{ }^{t} X & { }^{t} Y \\
-{ }^{t} Y & { }^{t} X
\end{array}\right] \cdot{ }^{t} S\right)
$$

Similarly one computes $\operatorname{Ad}_{z}\left(F_{i j}^{\prime}\right)$. We want to write $\operatorname{Ad}_{z}\left(E_{i j}^{\prime}\right)$ and $\operatorname{Ad}_{z}\left(F_{i j}^{\prime}\right)$ as linear combinations in $\left(E_{i j}^{\prime}, F_{i j}^{\prime}\right)$. For this we define $3 \times 3$ matrices $J_{i j}$ by the formula:

$$
J_{i j}=\varepsilon_{j}^{i}-\varepsilon_{i}^{j} \in o(3), \quad 1 \leqslant i<j \leqslant 3 .
$$

Note that

$$
{ }^{t} S \cdot E_{i j}^{\prime} \cdot S=\left[\begin{array}{cc}
J_{i j} & 0 \\
0 & -J_{i j}
\end{array}\right], \quad{ }^{t} S \cdot F_{i j}^{\prime} \cdot S=\left[\begin{array}{cc}
0 & J_{i j} \\
J_{i j} & 0
\end{array}\right] .
$$

Computations reveal that

$$
\begin{gathered}
\operatorname{Ad}(Z): E_{i j}^{\prime} \mapsto\left\{X J_{i j}^{t} X-Y J_{i j}{ }^{t} Y\right\}_{1}+\left\{X J_{i j}{ }^{t} Y+Y J_{i j}{ }^{t} X\right\}_{2}, \\
F_{i j}^{t} \mapsto\left\{-X J_{i j}{ }^{t} Y-Y J_{i j}{ }^{t} X\right\}_{1}+\left\{X J_{i j}{ }^{t} X-Y J_{i j}{ }^{t} Y\right\}_{2},
\end{gathered}
$$

where $\{\cdot\}_{1}$ means identify $J_{k \ell}$ with $E_{k \ell}^{\prime}$, and $\{\cdot\}_{2}$ means identify $J_{k \ell}$ with $F_{k \ell}^{\prime}$.

It is more convenient to rewrite the above using complex notation. So we write

$$
Z=\left(x_{j}^{i}+\sqrt{-1} y_{j}^{i}\right)=\left(z_{j}^{i}\right) \in U(3)
$$

and compute $\rho(Z)$ with respect to the complex basis

$$
E_{12}^{\prime}+i F_{12}^{\prime}, E_{13}^{\prime}+i F_{13}^{\prime}, E_{23}^{\prime}+i F_{23}^{\prime} \text {, }
$$

so that $\rho(Z) \in G L(3, \mathbb{C})$. (In other words, we have chosen an identification of $m$ with $\mathbb{C}^{3}$.) We find that

$$
\rho(Z)=\left[\begin{array}{lll}
Z_{33} & Z_{32} & Z_{31} \\
Z_{23} & Z_{22} & Z_{21} \\
Z_{13} & Z_{12} & Z_{11}
\end{array}\right] \in G L(3, \mathbb{C}),
$$

where $Z_{i j}$ denotes the $(i, j)$-minor of $Z$. We see that $\rho(Z)$ is related to the adjoint matrix of $Z$ in a simple way. (The adjoint matrix of $Z$ is the transpose of the cofactor matrix of $Z$ so that $Z$ times its adjoint matrix is the determinant of $Z$.) More precisely, we let

$$
\delta=\left[\begin{array}{lll} 
& & 1 \\
& -1 &
\end{array}\right]
$$


Then

$$
\rho(Z)=\operatorname{det}(Z) \delta \cdot \bar{Z} \cdot \delta \in G L(3, \mathbb{C}) .
$$

REMARK. More generally, consider the linear isotropy representation

$$
\rho: U(n) \rightarrow G L(N, \mathbb{C})
$$

where $\mathfrak{m}$ is identified with $\mathbb{C}^{N}, N=n(n-1) / 2$, via the lexicographical ordering of the root basis as in the $S O(6) / U(3)$ case. Identify $\mathbb{C}^{N}$ with $\Lambda^{2}\left(\mathbb{C}^{n}\right)$, the space of 2-vectors in $\mathbb{C}^{n}$. Calculations then show that

$$
\rho(Z)\left(\varepsilon_{i} \wedge \varepsilon_{j}\right)=Z_{i} \wedge Z_{j},
$$

where $Z=\left(Z_{i}\right) \in U(n)$, and $\left(\varepsilon_{i}\right)$ are the canonical basis vectors of $\mathbb{C}^{n}$. Extending linearly over the basis $\left\{\varepsilon_{i} \wedge \varepsilon_{j}: 1 \leqslant i<j \leqslant n\right\}$ of $\mathbb{C}^{N}$ one obtains the matrix representation $\rho$. However, the orbit structure of this action is very complicated for large $n$.

\section{The Frenet frame along a holomorphic CURVE}

Let $M$ be a Riemann surface and consider a holomorphic map

$$
f: M \rightarrow S O(6) / H .
$$

We let $e: U \subset M \rightarrow S O(6)$ denote a smooth local section of the $U(3)$-principal bundle

$$
f^{-1} S O(6) \rightarrow M .
$$

The holomorphy of $f$ is reflected by the fact that the forms

$$
e^{*} \Theta^{\prime i j}, \quad 1 \leqslant i<j \leqslant 3,
$$

are all of type $(1,0)$ on $M$.

IndeX CONVENTION. (12) $=1,(13)=2,(23)=3 ; 1 \leqslant i, j \leqslant 3,1 \leqslant \alpha, \beta \leqslant 6$.

Fix a Riemannian metric on $M$ from its conformal class, say $d s_{M}^{2}$. This means that we can locally write

$$
d s_{M}^{2}=\varphi \cdot \bar{\varphi}
$$

for some type $(1,0)$ nonvanishing form $\varphi$. The 1 -form $\varphi$ is called a unitary coframe.

We define complex valued local functions $\left(Z^{i}\right)$ on $M$ by

$$
e^{*} \Theta^{\prime i}=Z^{i} \varphi
$$


where $\varphi$ is a fixed unitary coframe.

Put $\tau_{1}=\sum\left|Z^{i}\right|^{2}$. It is routinely verified that $\tau_{1}$ is a globally defined smooth function on $M$. Let $\sum_{1}$ denote the zero set of $\tau_{1}$; we shall mostly work away from the set $\sum_{1}$.

Since $f$ is nonconstant, the function $\tau_{1}$ is not identically zero: since the pullback of the standard metric on $S O(6) / U(3)$ is given by

$$
d s_{1}^{2}=\tau_{1} \varphi \cdot \bar{\varphi},
$$

we see that $\tau_{1}(x)=0$ if and only if $x \in M$ is a non-immersion point. In fact, we can say a lot more. But first we need to recall the notion of an analytic type function.

DEFINITION: Let $U$ be a domain in the Riemann surface $M$. A $\mathbb{C}^{n}$-valued smooth function $h=\left(h^{i}\right)$ on $U$ is said to be of analytic type if for each point $x \in U$, if $z$ is a local holomorphic coordinate centred at $x$, then

$$
h=z^{b} \tilde{h},
$$

where $b$ is a positive integer and $\tilde{h}$ is a smooth $\mathbb{C}^{n}$-valued function with $\tilde{h}(x) \neq 0$.

So if $h$ is a function of analytic type on $U$, then $h$ is either identically zero or its zeros are isolated and of finite multiplicity (the integer $b$ in the above definition).

It is known [1] that the functions of analytic type are exactly solutions of exterior equation

$$
d h=\Phi h(\bmod \varphi),
$$

where $\Phi$ is an $n \times n$ matrix of complex valued 1-forms on $U$ and $\varphi$ is a nowhere zero type $(1,0)$ form on $U$.

Proposition. The function $\tau_{1}: M \rightarrow \mathbb{R}$ is an analytic type function on $M$.

PRoof: We will show that the local function

$$
\left(Z^{i}\right): U \subset M \rightarrow \mathbb{C}^{3}
$$

is of analytic type. Since $\tau_{1}=\sum\left|Z^{i}\right|^{2}$, the rest follows. Exterior differentiation of both sides of the equations

leads to

$$
\begin{gathered}
e^{*} \Theta^{\prime} i=Z^{i} \varphi \\
d Z^{i} \equiv \Psi_{j}^{i} Z^{j}(\bmod \varphi):
\end{gathered}
$$

one uses the Maurer-Cartan structure equations of $S O(6)$ and the equation

$$
d \varphi=-\theta_{C} \wedge \varphi,
$$


where $\theta_{C}$ is the complex connection form. For example,

$$
d Z^{1} \equiv\left(\theta_{C}+i \omega_{2}^{1}+i \omega_{4}^{3}\right) Z^{1}+\left(i \omega_{6}^{3}-\omega_{6}^{4}\right) Z^{2}+\left(\omega_{6}^{2}-i \omega_{6}^{1}\right) Z^{3}(\bmod \varphi),
$$

where $\omega=e^{*} \Omega$.

Note that $r_{1}=\sqrt{\tau_{1}}$ is a continuous function on $M$ smooth away from its zeros.

Suppose $\tilde{e}: \tilde{U} \rightarrow S O(6)$ is another lifting of $f$. This means that

$$
\tilde{e}=e \cdot k,
$$

for some smooth function $k: U \cap \tilde{U} \rightarrow U(3)$. Define $\left(\tilde{Z}^{i}\right)$ by setting

$$
\tilde{e}^{*} \Theta^{\prime i}=\tilde{Z}^{i} \varphi
$$

We then obtain the following

TRANSFORMATION RULE. $\left(\tilde{Z}^{i}\right)=\rho\left(k^{-1}\right)\left(Z^{i}\right)$.

The above rule follows from the fact that

$$
(e \cdot k)^{*} \Omega_{\mathfrak{m}}=\operatorname{Ad}\left(k^{-1}\right) e^{*} \Omega_{\mathfrak{m}}
$$

Consulting $\left(^{*}\right)$ in the preceding section it is clear that near a point in $M \backslash \sum_{1}$ we can make

$$
Z^{1}=r_{1}, \quad Z^{2}=Z^{3}=0
$$

where $r_{1}=\sqrt{\tau_{1}}$. Any lifting $e$ achieving this will be called a first order frame.

Let $e$ be any first order frame along $f$, and write $\omega=e^{*} \Omega$. We then have

$$
\begin{gathered}
\frac{1}{2}\left[\left(\omega_{3}^{1}-\omega_{4}^{2}\right)+i\left(\omega_{3}^{2}+\omega_{4}^{1}\right)\right]=r_{1} \varphi, \\
\omega_{5}^{1}=\omega_{6}^{2}, \omega_{5}^{2}=-\omega_{6}^{1}, \\
\omega_{5}^{3}=\omega_{6}^{4}, \omega_{5}^{4}=\omega_{6}^{3} .
\end{gathered}
$$

Define a subgroup of $U(3)$ by

$$
G_{1}=\left\{k \in U(3): \rho(k)^{t}(1,0,0)={ }^{t}(1,0,0)\right\}
$$

We find that

$$
G_{1}=\left\{\left[\begin{array}{cc}
Z & 0 \\
0 & \exp (i \theta)
\end{array}\right]: Z \in S U(2)\right\} \cong S U(2) \times U(1)
$$


Let $e$ be a first order frame along $f$. Then any other first order frame along $f$ is given by $e \cdot k$, where $k$ is a $G_{1}$-valued local function on $M$.

The first order frames are constructed near a point $x \in M \backslash \sum_{1}$. Near a point in $\sum_{1}$ we can find a generalised first order frame e such that

$$
e^{*} \Theta^{\prime 2}=e^{*} \Theta^{\prime 3}=0, \quad e^{*} \Theta^{\prime 1}=Z_{1} \varphi, Z_{1} \text { complex valued, }
$$

where $\left|Z_{1}\right|^{2}=\tau_{1}$. To see this observe that $\left(Z^{i}\right)$ can be written as

$$
z^{b}\left(\widehat{Z}^{i}\right)
$$

where $z$ is a local holomorphic coordinate centred at $x$ and $\left(\widehat{Z}^{i}(x)\right) \neq 0$. Thus we can use the $\boldsymbol{H}$-action to bring about

$$
\widehat{Z}^{1}=\widehat{r}_{1}>0, \widehat{Z}^{2}=\widehat{Z}^{3}=0 .
$$

Then $Z_{1}=z^{b} \hat{\boldsymbol{r}}^{1}$ does the trick.

By way of notation we put

$$
\psi^{1}=\frac{1}{2}\left(\omega_{3}^{1}-\omega_{4}^{2}\right), \psi^{2}=\frac{1}{2}\left(\omega_{3}^{2}+\omega_{4}^{1}\right), \psi=\psi^{1}+i \psi^{2} .
$$

Note that $\psi$ is of type $(1,0)$, and that

$$
\psi=Z_{1} \varphi
$$

and near a point in $M \backslash \Sigma_{1}$

$$
\psi=r_{1} \varphi
$$

We now exterior differentiate the relations $(\mathrm{E} 1-3)$ and construct the second order frames. We will use the Maurer-Cartan structure equations of $S O(6)$. We will also need the structure equations for $(M, \varphi \cdot \bar{\varphi})$ :

$$
d \varphi=-\theta_{C} \wedge \varphi
$$

The purely imaginary 1-form $\theta_{C}$ is the complex connection form with respect to $\varphi$, and is nothing but $-i$ times the Levi-Civita connection form of $\left(M,\left(\varphi^{i}\right)\right)$. Differentiating this equation one more time we obtain

$$
d \theta_{C}=\frac{K}{2} \varphi \wedge \bar{\varphi}, \quad K=\text { the Gaussian curvature. }
$$


We now exterior differentiate the left hand side of (E1):

$$
d\left(\psi^{1}+i \psi^{2}\right)=i\left(\omega_{2}^{1}+\omega_{4}^{3}\right) \wedge\left(\psi^{1}+i \psi^{2}\right)=i\left(\omega_{2}^{1}+\omega_{4}^{3}\right) \wedge r_{1} \varphi
$$

On the other hand,

$$
d\left(r_{1} \varphi\right)=d r_{1} \wedge \varphi-r_{1} \theta_{C} \wedge \varphi=\left(d r_{1}-r_{1} \theta_{C}\right) \wedge \varphi
$$

It follows that

$$
\left[d \log r_{1}-i\left(\omega_{2}^{1}+\omega_{1}^{3}\right)-\theta_{C}\right] \wedge \varphi=0 .
$$

Since $d \log r_{1}$ is real and $i\left(\omega_{2}^{1}+\omega_{4}^{3}\right)+\theta_{C}$ is purely imaginary, we then must have

$$
* d \log r_{1}=i\left[\theta_{C}+i\left(\omega_{2}^{1}+\omega_{4}^{3}\right)\right]
$$

where * is the Hodge operator of $\left(M, d s^{2}\right)$.

Exterior differentiation of the first equation in (E2) leads to

$$
\omega_{5}^{3} \wedge \varphi^{1}+\omega_{5}^{4} \wedge \varphi^{2}=0
$$

the second equation yields

$$
\omega_{5}^{4} \wedge \varphi^{1}-\omega_{5}^{3} \wedge \varphi^{2}=0
$$

It follows from $(t, t)$ that

$$
\omega_{5}^{4}=-* \omega_{5}^{3}, \quad \omega_{5}^{2}=-* \omega_{5}^{1},
$$

and we may set

$$
\omega_{5}^{1}-i \omega_{5}^{2}=Z^{1} \varphi, \quad \omega_{5}^{3}-i \omega_{5}^{4}=Z^{2} \varphi
$$

for some local complex valued functions $\left(Z^{i}\right)$ on $M$.

Put $\tau_{2}=\sum\left|Z^{i}\right|^{2}$. It is easily verified that $\tau_{2}$ is a well-defined smooth function on $M$. A consideration similar to that given for $\tau_{1}$ shows that $\tau_{2}$ is an analytic type function on $M$. Let $\sum_{2}$ denote the zero set of $\tau_{2}$.

Define tilded quantities $\widetilde{Z}^{1}, \widetilde{Z}^{2}$ by

$$
\tilde{\omega}_{5}^{1}-i \tilde{\omega}_{5}^{2}=\tilde{Z}^{1} \varphi, \quad \tilde{\omega}_{5}^{3}-i \tilde{\omega}_{5}^{4}=\tilde{Z}^{2} \varphi, \quad \tilde{\omega}=\tilde{e}^{*} \Omega,
$$

where $\tilde{e}=e \cdot k, k \in G_{1}$-valued, is another first order frame. Write

$$
k=\left(Z, e^{i \theta}\right)
$$


where $Z$ is $S U(2)$-valued. We can write $Z$ as

$$
Z=\left[\begin{array}{cc}
z_{1}^{1} & z_{2}^{1} \\
-\bar{Z}_{2}^{1} & \bar{z}_{1}^{1}
\end{array}\right], \quad z_{j}^{i}=x_{j}^{i}+i y_{j}^{i}
$$

From the formula $\tilde{\omega}=i\left(k^{-1}\right) \cdot \omega \cdot i(k)$ we compute that

$$
{ }^{t}\left(\tilde{Z}^{i}\right)=e^{-i \theta} \cdot{ }^{t} Z \cdot{ }^{t}\left(Z^{i}\right) .
$$

We see from this that we can make $Z^{1}=r_{2}>0$, and $Z^{2}=0$.

Summarising the preceding computation, we have

Proposition. Let $f: M \rightarrow S O(6) / U(3)$ be a nonconstant holomorphic map. Near any point $x \in M \backslash\left\{\sum_{1} \cup \sum_{2}\right\}$ there exists a local lifting $e$ into $S O(6)$ such that in addition to $(E 1-3)$ we have

$$
\omega_{5}^{1}-i \omega_{5}^{2}=r_{2} \varphi, \quad \omega_{5}^{3}=\omega_{5}^{4}=0,
$$

where, as usual, $\omega=e^{*} \Omega$.

The totality of such frames, called the second order frames, is determined up to the structure group

$$
\begin{aligned}
G_{2} & =\left\{\left(Z, e^{i \theta}\right) \in S U(2) \times U(1): e^{-i \theta} \cdot{ }^{t} Z \cdot{ }^{t}(1,0)={ }^{t}(1,0)\right\} \\
& =\left\{\left(e^{i \theta}, e^{-i \theta}, e^{i \theta}\right) \in U(3)\right\} \cong U(1) .
\end{aligned}
$$

THEOREM. Suppose $\tau_{2}(f) \equiv 0$. Then $f(M)$ is congruent to an open submanifold of $S O(4) / U(2) \cong \mathbb{C} P^{1}$.

Proof: $\tau_{2}(f) \equiv 0$ means that the bundle of first order frames along $f$, denoted by $L_{1}$, is an integral manifold of the exterior system

$$
\Omega_{B}^{A}=0, \quad 1 \leqslant A \leqslant 4, \quad 5 \leqslant B \leqslant 6,
$$

on $S O(6)$. (So a first order frame along $f$ is a local section of $L_{1} \rightarrow M$.) It follows that $L_{1}$ is a translate of $S O(4) \times S O(2) \subset S O(6)$. Then $f(M)$ is congruent to a submanifold of

$$
S O(4) \times S O(2) /(U(3) \cap S O(4) \times S O(2)) \cong S O(4) / U(2) \cong \mathbb{C} P^{1} .
$$

Hereafter we assume that $\tau_{2}$ is not identically zero.

We now exterior differentiate both sides of the equations in (E4), and construct the third order frames. 
We obtain from the first equation of (E4)

$$
\begin{gathered}
d\left(\omega_{5}^{1}-i \omega_{5}^{2}\right)=i\left(\omega_{6}^{5}-\omega_{2}^{1}\right) \wedge r_{2} \varphi \\
d\left(r_{2} \varphi\right)=d r_{2} \wedge \varphi-r_{2} \theta_{C} \wedge \varphi
\end{gathered}
$$

Consequently,

$$
\left\{d \log r_{2}-\theta_{C}-i\left(\omega_{6}^{5}-\omega_{2}^{1}\right)\right\} \wedge \varphi=0
$$

Therefore

$$
* d \log r_{2}=i\left(\theta_{C}+i\left(\omega_{6}^{5}-\omega_{2}^{1}\right)\right)
$$

The remaining two equations in (E4), upon exterior differentiation, yield

$$
\omega_{3}^{1} \wedge \varphi^{1}-\omega_{3}^{2} \wedge \varphi^{2}=0, \quad \omega_{4}^{1} \wedge \varphi^{1}-\omega_{4}^{2} \wedge \varphi^{2}=0 .
$$

Consequently, we can write

$$
\begin{aligned}
\omega_{3}^{1}=a \varphi^{1}+b \varphi^{2}, & \omega_{4}^{1}=b \varphi^{1}+c \varphi^{2}, \\
\omega_{3}^{2}=* \omega_{3}^{1}, & \omega_{4}^{2}=* \omega_{4}^{1},
\end{aligned}
$$

where $a, b, c$ are some local functions on $M$ with $a+c=2 r_{1}$.

Define tilded quantities $\tilde{a}, \tilde{b}, \tilde{c}$ using another second order frame $\tilde{e}=e \cdot k$, where

$$
k=\left(e^{i \theta}, e^{-i \theta}, e^{i \theta}\right), \quad \theta \text { a local function on } M
$$

We want to know how $(\tilde{a}, \tilde{b}, \tilde{c})$ are related to $(a, b, c)$.

Again using the formula

$$
\widetilde{\omega}=i\left(k^{-1}\right) \cdot \omega \cdot i(k), \omega=e^{*} \Omega, \tilde{\omega}=\widetilde{e}^{*} \Omega
$$

we compute that

$$
\begin{aligned}
& \tilde{a}=a \cdot \cos ^{2} \theta+c \cdot \sin ^{2} \theta-2 b \cdot \cos \theta \sin \theta \\
& \tilde{b}=b \cdot \cos 2 \theta+(a-c) \cdot \cos \theta \sin \theta \\
& \tilde{c}=a \cdot \sin ^{2} \theta+c \cdot \cos ^{2} \theta+b \cdot \sin 2 \theta
\end{aligned}
$$

If $b$ does not vanish, then we can smoothly choose $\theta$ so that

$$
\operatorname{cotan} 2 \theta=(c-a) / 2 b
$$

making $\tilde{b}=0$. All this leads to the third, and final, normal form

$$
\omega_{3}^{1}+i \omega_{3}^{2}=a \varphi, \quad \omega_{4}^{1}+i \omega_{4}^{2}=-i c \varphi,
$$


where $a+c=2 r_{1}$. Put $r_{3}=(a-c) / 2$.

The function $r_{3}=r_{3}^{2}$ is an analytic type function on $M$; we let $\sum_{3}$ denote the zero set of $\tau_{\mathbf{3}}$.

The isotropy group $G_{3}$ is given by

$$
G_{3}=\left\{\left(e^{i \theta}, e^{-i \theta}, e^{i \theta}\right) \in G_{2}: \theta=n \pi / 2, n \in \mathbb{Z}\right\} \cong \mathbb{Z}_{4} .
$$

It follows that near a point $x \in M \backslash\left\{\sum_{1} \cup \sum_{2} \cup \sum_{3}\right\}$ there is a more or less unique lifting

$$
e_{f}: U \subset M \rightarrow S O(6)
$$

achieving the normal forms (E1) through (E5). Such a lifting will be called a Frenet frame along $f$.

Exterior differentiation of both sides of the equations in (E5) leads to

$$
\begin{aligned}
& * d \log r_{1}=i\left(\theta_{C}+i\left(\omega_{2}^{1}+\omega_{4}^{3}\right)\right), \\
& * d \log r_{3}=i\left(\theta_{C}+i\left(\omega_{2}^{1}-\omega_{4}^{3}\right)\right) .
\end{aligned}
$$

Remark. Suppose $\tau_{3}(f)$ is identically zero. Then one can show that $f(M)$ lies in the image of a $\mathbb{C} P^{2} \subset \mathbb{C} P^{3}$ under the symmetric space isomorphism

$$
C P^{3} \cong S O(6) / U(3) \text {. }
$$

THEOREM. Let $f: M \rightarrow S O(6) / U(3)$ be a nonconstant holomorphic map. Fix a conformal metric $d s^{2}=\varphi \cdot \bar{\varphi}, \varphi \in$ type $(1,0)$, and define the differential invariants $\left(r_{i}\right)$ as in the above. We then have

$$
\begin{aligned}
& \Delta \log r_{1}=K-4 \tau_{1}+2 \tau_{2}, \\
& \Delta \log r_{2}=K+2\left(\tau_{1}+\tau_{3}\right)-4 \tau_{2}, \\
& \Delta \log r_{3}=K+2 \tau_{2}-4 \tau_{3},
\end{aligned}
$$

away from the singular locus $\sum_{1} \cup \sum_{2} \cup \sum_{3} \subset M$.

Proof: Exterior differentiate both sides of the equations in (F1 - 3) using

$$
\begin{aligned}
d * d \log r_{i} & =\frac{i}{2} \Delta \log r_{i} \varphi \wedge \bar{\varphi} \\
d \omega_{2}^{1} & =\frac{i}{2}\left(a^{2}+c^{2}-2 r_{2}^{2}\right) \varphi \wedge \bar{\varphi} \\
d \omega_{4}^{3} & =i a c \varphi \wedge \bar{\varphi} \\
d \omega_{8}^{5} & =i r_{2}^{2} \varphi \wedge \bar{\varphi}
\end{aligned}
$$

We give an application. 
Corollary. Suppose $f: M \rightarrow S O(6) / U(3)$ is a holomorphic isometric immersion from a compact $M$. Further suppose that $K \geqslant 4 / 3$, where $K$ is the Gaussian curvature of $\left(M, d s^{2}\right)$. Then we must have $K=4 / 3$.

Proof: $K \geqslant 4 / 3$ implies that

$$
\Delta \log \left(r_{2}^{2} r_{3}\right) \geqslant 0 .
$$

Thus $\log \left(r_{2}^{2} r_{3}\right)$ is a subharmonic function with singularties at the zeros of $r_{2}$ and $r_{3}$ where it goes to $-\infty$. In particular, this function attains a maximum on $M$. Now the maximum principle for subharmonic functions says that it must be a constant.

\section{The integrability conditions and the Associated PDE system}

In this section we summarise the frame construction by setting up a bijective correspondence between the holomorphic curves in $S O(6) / U(3)$ and the solutions to the PDE system coming from (I1 - 3).

Definition: We shall say that $f: M \rightarrow S O(6) / U(3)$ is a nondegenerate curve if none of the $\tau_{i}$ 's are identically zero. The map $f$ will be called a regular curve if $\tau_{1} \cdot \tau_{2} \cdot \tau_{3}$ is never zero.

Observe that the regularity assumption is a global assumption.

Consider the following exterior differential system, denoted by $\mathcal{S}$, defined on $M \times$ $S O(6)$ with independence condition $\varphi \wedge \bar{\varphi} \neq 0$ :

$$
\begin{aligned}
\Omega_{3}^{1}+i \Omega_{3}^{2}= & \left(r_{1}+r_{3}\right) \varphi,-\Omega_{4}^{2}+i \Omega_{4}^{1}=\left(r_{1}-r_{3}\right) \varphi, \\
& \Omega_{5}^{1}-i \Omega_{5}^{2}=\Omega_{6}^{2}-i \Omega_{6}^{1}=r_{2} \varphi, \\
& \Omega_{5}^{3}=\Omega_{5}^{4}=\Omega_{6}^{4}=\Omega_{6}^{3}=0 . \\
& \Omega_{2}^{1}+\Omega_{4}^{3}=i \theta_{C}-* d \log r_{1}, \\
& \Omega_{6}^{5}-\Omega_{2}^{1}=i \theta_{C}-* d \log r_{2}, \\
& \Omega_{2}^{1}-\Omega_{4}^{3}=i \theta_{C}-* d \log r_{3},
\end{aligned}
$$

where $\theta_{C}$ is the complex connection form of $(M, \varphi \cdot \bar{\varphi})$, and the $r_{i}$ 's are any positive functions on $M$ solving the PDE system

$$
\begin{aligned}
& \Delta \log r_{1}=K-4 r_{1}^{2}+2 r_{2}^{2}, \\
& \Delta \log r_{2}=K+2\left(r_{1}^{2}+r_{3}^{2}\right)-4 r_{2}^{2}, \\
& \Delta \log r_{3}=K+2 r_{2}^{2}-2 r_{3}^{2} .
\end{aligned}
$$

THEOREM. The set of regular holomorphic curves $M \rightarrow S O(6) / U(3)$ is in bijective correspondence with the set of all solutions $\left(r_{1}, r_{2}, r_{3}\right)$ to the integrability conditions (I1 -3). 
Proof: Any regular curve certainly gives rise to such a solution: this is the content of the frame construction given in the preceding section. Conversely suppose we are given such a solution $\left(r_{i}\right)$. Counting the number of independent equations in $\mathcal{S}$ we see that $\mathcal{S}$ defines a two-dimensional distribution on $M \times S O(6)$. Moreover, this distribution is completely integrable and, hence, defines a foliation on $M \times S O(6)$. The independence condition $\varphi \wedge \bar{\varphi} \neq 0$ implies that a leaf of this foliation can be written locally as

$$
U \rightarrow U \times S O(6), \quad z \mapsto(z, e(z)) .
$$

It is straightforward to verify that $e(z)$ is a Frenet frame along $f=\pi \circ e$, where $\pi$ denotes the projection $S O(6) \rightarrow S O(6) / U(3)$.

\section{Compact curves}

In this section we give the integrated version of the integrability conditions (I1 3) assuming that $M$ is compact.

Definition: Let $M$ be a Riemann surface. A singular Hermitian metric on $M$ is given locally as

$$
d s^{2}=\psi \cdot \bar{\psi},
$$

where $\psi$ is a type $(1,0)$ smooth form of analytic type, that is, $\psi$ can be written as the product of an analytic type function and a nowhere vanishing type $(1,0)$ form. We can rewrite $d s^{2}$ as

$$
d s^{2}=h(z) d z \cdot d \bar{z},
$$

where $h(z) \geqslant 0$ and $z$ is a holomorphic coordinate. Moreover, we have

$$
h(z)=|z|^{2 n} \tilde{h}(z),
$$

where $\widetilde{h}(z)$ is never zero and $n$ is a nonnegative integer. The integer $n$ is the order of $\psi$ at $z=0$ and we write $\operatorname{ord}_{0} \psi=n$. The singular divisor of $d s^{2}$, denoted by $D_{\psi}$, is defined to be the zero divisor of $\psi$. So

$$
D_{\psi}=\sum \operatorname{ord}_{p}(\varphi) p, p \in M .
$$

It is easy to see that $D_{\psi}$ depends only on the singular metric, not on the particular choice of $\psi$. The degree of $D_{\psi}$ is locally finite, and is the total number of zeros of $\psi$ counted with multiplicity.

Given a singular metric $d s^{2}$ on $M$ we have the usual Hermitian structure equations away from the support of the singular divisor:

$$
d \psi=-\theta_{C} \wedge \psi, d \theta_{C}=\frac{K}{2} \psi \wedge \bar{\psi}=(-i K) \cdot \text { the Kähler form. }
$$

There is the 
Generalised Gauss-Bonnet-Chern Theorem. Let $M$ be a compact Riemann surface of genus $g$ equipped with a singular metric $\psi \bar{\psi}$. Then

$$
\frac{i}{2 \pi} \int_{M} d \theta_{C}=2-2 g+\operatorname{deg} D_{\psi}
$$

Proof: This follows from the usual Gauss-Bonnet-Chern theorem combined with the argument principle: one notes that $d \theta_{C}$ is a multiple of $\Delta \log h(d z \wedge d \bar{z})$.

Given a nondegenerate holomorphic curve $f: M \rightarrow S O(6) / U(3)$ we define the $i$ th osculating metric to be

$$
d s_{i}^{2}=\tau_{i} \varphi \cdot \bar{\varphi} .
$$

These metrics are singular metrics. (Note that $d s_{1}^{2}$ is just the induced metric.) We put

$$
\begin{gathered}
\varphi_{i}=r_{i} \varphi \\
\Lambda_{i}=\frac{i}{2} \varphi_{i} \wedge \bar{\varphi}_{i}=\text { the Kähler form of }\left(M, d s_{i}^{2}\right), \\
\theta_{i, C}=\text { the complex connection form of }\left(M, d s_{i}^{2}\right), \\
K_{i}=\text { the Gaussian curvature of }\left(M, d s_{i}^{2}\right)
\end{gathered}
$$

so that

$$
d \varphi_{i}=-\theta_{i, c} \wedge \varphi_{i}, d \theta_{i, c}=-i K_{i} \Lambda_{i} .
$$

Let $e_{f}$ be a Frenet frame along $f$, and put $\omega=e^{*} \Omega$. Consulting the normal forms $(E 1-5)$ in Section 3 we compute that

$$
\begin{aligned}
& \theta_{1, C}=i\left(\omega_{1}^{2}+\omega_{3}^{4}\right), \\
& \theta_{2, C}=i\left(\omega_{5}^{0}-\omega_{1}^{2}\right), \\
& \theta_{3, C}=i\left(\omega_{1}^{2}-\omega_{3}^{4}\right) .
\end{aligned}
$$

(For example, the first and third relations follow upon exterior differentiating the first two equations in (E5).) Exterior differentiation of these equations leads to

$$
\begin{aligned}
& d \theta_{1, c}=2 i\left(-2 \Lambda_{1}+\Lambda_{2}\right) \\
& d \theta_{2, c}=2 i\left(\Lambda_{1}-2 \Lambda_{2}+\Lambda_{3}\right) \\
& d \theta_{3, C}=2 i\left(\Lambda_{2}-2 \Lambda_{3}\right)
\end{aligned}
$$

TheOREM. Let $M=M_{g}$ denote a compact Riemann surface of genus $g$, and consider a nondegenerate curve $f: M \rightarrow S O(6) / U(3)$. Then

$$
\begin{gathered}
2 g-2-\#_{i}=d_{i-1}-2 d_{i}+d_{i+1}, \\
\#_{i}=\operatorname{deg} D_{\varphi i}=\text { the total number of zeros of } r_{i} \\
d_{i}=\frac{1}{\pi} \cdot\left(\begin{array}{c}
\text { the area of } \left.\left(M, d s_{i}^{2}\right)\right) \\
d_{-1}=d_{4}=0 .
\end{array}\right.
\end{gathered}
$$

where 
Proof: We have

$$
\frac{i}{2 \pi} \int_{M} d \theta_{i, c}=2-2 g+\#_{i}
$$

from the generalised Gauss-Bonnet-Chern theorem. From the Wirtinger theorem

$$
\int_{M} \Lambda_{i}=\text { the area of }\left(M, d s_{i}^{2}\right),
$$

and the result follows.

REmark. The relations in (P) correspond to the Plucker relations for algebraic curves in $\mathbb{C} P^{3}[2]$ p.86-95).

\section{REFERENCES}

[1] S. Chern, 'On the minimal immersions of the two-sphere in a space of constant curvature', in Problems in analysis, pp. 27-40 (Princeton Univ. Press, 1970).

[2] K. Yang, Complete and compact minimal surfaces (Kluwer Academic Publishers, Boston, 1989).

Department of Mathematics

Arkansas State University

State University, Arkansas 72467

United States of America 